\title{
Essai d'estimation du coefficient d'utilisation de la biomasse herbacée par le bétail dans un périmètre sahélien
}

\author{
par B. TOUTAIN $\left(^{*}\right)$ et P. LHOSTE $\left({ }^{*}\right)$
}

\begin{abstract}
RẼSUMÉ
Dans une petite région sahélienne bien délımitée, les auteurs essalent d'estimer le coefficient d'utilisation moyen de la biomasse v'gétale herbacée appétible par l'ensemble du bétail présent au cours de l'annee, en comparant l'évaluation des réserves fourragères sur l'ensemble du périmètre et l'évaluation des besoins fourragers du bétail estimés après comptage des animaux domestiques herbivores fréquentant cette région. Les observations recueillies, permettent de situer ce coefficient à environ 35 p. 100.
\end{abstract}

Les pâturages naturels des grandes steppes sahéliennes sont constitués en majorité de graminées annuelles disposées en tapis plus ou moins continu. Les pailles sur pied de ces graminées représentent la principale réserve fourragère pour les quelque 9 mois de saison sèche.

La quantité de fourrage ainsi disponible peut être estimée assez aisément à partir de mesures de la biomasse aérienne herbacée en début de saison sèche. En revanche, la proportion de ce potentiel fourrager qui sera effectivement ingérée par les animaux et ensuite métabolisée n'est pas bien connue. La connaissance de ce coefficient d'utilisation des disponibilités fourragères dans les conditions de l'élevage extensif sahélien est nécessaire pour évaluer la capacité de charge d'un pâturage ou pour connaître l'effectif animal optimal d'une parcelle ou d'une zone déterminée.

La valeur communément employée de ce coefficient d'utilisation des disponibilités four-

(*) Institut d'Elevage et de Médecine Vétérinaire des Pays Tropicaux, 10, rue Pierre-Curie, 94700 MasonsAlfort.

(**) C. R. Z, de Minanko, B. P. 1152, Bouaké (Côted'Ivoire) et I. E. M. V. T. (voir ci-dessus). ragères a été établie de façon théorique. Des mesures périodiques de la biomasse herbacéedurant la saison sèche ont révélé (2) que la moitié de cette biomasse se trouvait progressivement dégradée et perdue au cours des 9 mois secs (pailles brisées par le vent, action des termites...). L'observation des animaux au pâturage révèle que le broutage est très dispersé et laisse d'abondants refus : la part prélevée à chaque passage de troupeau ne représente jamais qu'une fraction de la masse végétale présente. A cette part prélevée s'ajoute ce qui est piétiné et perdu. De plus, il convient qu'un certain couvert végétal demeure au moment des premières tornades pour protéger le sol de l'effet érosif du vent, des gouttes de pluie et du ruissellement, et aussi pour maintenir au sol le tapis de semences qui assurera le renouvellement du tapis végétal annuel. Finalement, on estime que la consommation effective de fourrage par les animaux, dans les conditions d'exploitation qui permettent la pérennité du pâturage, représente $1 / 3$ au maximum de la biomasse herbacée appétible présente au début de la saison sèche.

Dans le cadre d'une action complémentaire concertée sur le thème de la lutte contre l'aridité en zone sahélienne organisée et financée 
par la D. G. R. S. T., deux études ont été réalisées simultanément sur le même périmètre, dans la région de Gorom-Gorom et Oursi en Haute-Volta :

- l'étude zootechnique, comprenant le dénombrement et la caractérisation des troupeaux (5) ;

- l'étude et la cartographie des ressources pastorales, avec notamment l'évolution des productivités des pâturages (6).

Le but du présent travail est de comparer l'effectif des animaux aux ressources pastorales dont ils disposent, et d'apporter des précisions sur l'utilisation réelle par les troupeaux des disponibilités fourragères.

\section{MÉTHODE D'ÉTUDE}

\section{Périmètre de l'étude}

La région étudiée couvre une superficie approximative de 64000 ha. Elle est située dans l'Oudalan, province la plus sahélienne de HauteVolta. La période pluvieuse dure 3 mois, de la mi-juin à la mi-septembre, et à ce moment il tombe près de $400 \mathrm{~mm}$ de pluie. La saison sèche dure 9 mois. Elle est rigoureuse. La richesse essentielle de cette région est l'élevage. En outre, les meilleurs sols sont cultivés en mil et même en sorgho, mais avec des productions irrégulières et aléatoires. Les limites du périmètre correspondent à celles de la zone d'endodromie $\left(^{*}\right)$ Gorom-Gorom, Oursi, Bidi définie et étudiée par BARRAL (1). A l'intérieur de ces limites, les pratiques d'élevage se ressemblent, les troupeaux s'abreuvent aux mêmes points d'eau et transhument sur les mêmes parcours.

\section{Dénombrement des animaux}

L'évaluation des effectifs des animaux domestiques est un problème difficile chez les éleveurs sahéliens. Il est, en effet, exclu d'obtenir des chiffres d'effectif valables par enquête directe auprès de l'éleveur, propriétaire du bétail ou

(*) Une zone d'endodromie a été définie comme la zone exploitée en commun tout au long de l'année, à partir d'un ensemble de points d'eau permanents, par des éleveurs sédentajres ou nomades utilisant ces points d'eau en saison sèche, et ayant adopté les mêmes aires et le même calendrier de transhumance (1). berger. Les statistiques existantes, établies par le Service de l'Elevage ou à l'occasion de recensements officiels, sont difficilement utilisables, pour les mêmes raisons, telles quelles. Il est bien connu, en effet, que les déclarations des éleveurs à l'administration sont très inférieures à la réalité.

Dans ces conditions, pour approcher la réalité, nous avons exploité la contrainte d'abreuvement en fin de saison sèche ; celle-ci entraîne des concentrations d'animaux et des passages obligés des troupeaux sur les derniers points d'eau permanents. 11 y a donc lieu, tout d'abord, de recenser ces points d'abreuvement, avec précision, pour la zone d'étude considérée. L'évaluation primaire des effectifs sera alors fondée sur des observations et comptages en ces lieux de rassemblement : mares, puits, puisards... Ces comptages sont associés à une brève enquête à laquelle le berger accepte de répondre à savoir :

- Provenance du troupeau : emplacement du campement...

- Transhumance éventuelle: mouvements du troupeau...

- Rythme d'abreuvement : quotidien, tous les deux jours...

- Le troupeau est-il complet ?...

Outre ces comptages directs, nous avons également expérimenté la technique de photographie aérienne qui permet de dénombrer les animaux sur les points d'eau. Le principe en est de photographier, à l'occasion d'un survol en avion léger, tous les animaux rassemblés à proximité du point d'eau, à jour et heure donnés. Cela peut se faire en un ou plusieurs clichés suivant la dimension du point d'eau. La connaissance du fonctionnement et du rythme du point d'abreuvement, établie par enquête au sol, permet de connaître la proportion des animaux présents au jour et à l'heure donnés (estimation moyenne), et donc d'évaluer la population complète d'animaux s'abreuvant sur le point d'eau considéré.

Si les comptages directs ou sur photographies donnent des résultats assez bons pour les bovins, le dénombrement des petits ruminants est beaucoup plus difficile. Les ovins et caprins, qui ont une grande importance économique et sociale au Sahel, sont, en effet, plus mobiles et semblent avoir moins de régularité dans leur comportement et leurs habitudes : déplacements, abreuvement, lieu de repos... De plus, ils se présentent souvent sur les points d'eau en gros troupeaux 
serrés, ce qui rend tout comptage difficile et imprécis. Il n'est pas rare également que les chèvres soient abreuvées au puisard de nuit, à la saison chaude; les jeunes animaux sont le plus souvent abreuvés au campement, ce qui rend l'observation de troupeaux complets difficile. Il est donc nécessaire de recouper les résultats des dénombrements directs avec ceux obtenus par d'autres méthodes. Les rapports qui peuvent être établis, par sondage, entre les effectifs des différentes espèces domestiques, sont intéressants, à cet égard. Ces rapports sont également calculés, par ethnie, entre le nombre des membres de la famille et le nombre des animaux détenus. Ces rapports sont obtenus grâce à des comptages minutieux sur certains échantillons : campements, villages... Ils sont également confirmés par des comptages aléatoires et des sondages lors des déplacements ou à l'occasion d'observations diverses.

\section{Evaluation de la production fourragère}

Elle ne peut se faire qu'une fois l'étude et la cartographie des pâturages du périmètre achevées. L'étude commence par l'inventaire des différents types de pâturage, en référence à leur composition botanique et phytosociologique, et à la structure de la végétation. Chaque type de pâturage est en outre subdivisé en faciès selon l'état de la végétation. Les faciès sont définis selon certains indices de recouvrement de la végétation herbacée et de densité ligneuse résultant d'une éventuelle mortalité. Ces faciès, ainsi définis, sont à la fois reconnaissables sur le terrain et discernables sur les photographies aériennes. Ils correspondent à des classes de production de fourrage.

La cartographie des différents types de pâturages et de leurs faciès est réalisée par photointerprétation de la couverture photographique aérienne à $1 / 50000$. Les critères de photo-interprétation sont précisés au cours d'une mission sur le terrain.

Chaque faciès de chaque type de pâturage correspond à une classe de productivité du tapis herbacé appétible. Les mesures de la biomasse herbacée sont réalisées par coupe et pesée du tapis graminéen appétible sur une surface échantillon ( 9 à $25 \mathrm{~m}^{2}$ ), en début de saison sèche, au moment où s'achève la végétation des plantes annuelles. Le recouvrement du tapis est mesuré ou évalué à l'œil sur de grandes surfaces. On obtient ainsi, en combinant la productivité moyenne des plages couvertes et le taux de recouvrement dans des lieux bien caractéristiques, la détermination de la productivité du faciès de la formation. Enfin le planimétrage permet de connaître les surfaces de chaque type de formation et de chaque faciès.

Les principaux fourrages naturels font l'objet d'une analyse bromatologique qui permet d'en déterminer la valeur énergétique et fourragère. Cette dernière est exprimée en unités fourragères (U. F.) par kilogramme de matière sèche.

L'estimation de la production fourragère se heurte à plusieurs incertitudes :

- Les résidus de culture (feuilles et tiges de mil et de sorgho) représentent un fourrage dont le coefficient d'utilisation n'est pas forcément identique à celui d'un fourrage naturel. Cependant on considérera, comme cela paraît en première approximation, que les périmètres cultivés fournissent aux animaux la même quantité de fourrage effectivement consommé que la végétation naturelle avant le défrichement ;

- Le fourrage fourni par les ligneux (feuilles, fruits et gousses) n'entre pas en ligne de compte dans cette évaluation, alors que les animaux recourent à cette source de nourriture pendant une partie de l'année. La production de pâturage arbustif et la part consommée par les troupeaux sont mal connues. Ce fourrage représente un appoint riche en matières protéiques et en sels minéraux, au moment où les pailles de graminées en sont insuffisamment pourvues. Ce type de fourrage ne représentant qu'un complément à l'alimentation pouvant équilibrer la ration, il a été négligé dans le calcul de la production fourragère totale ;

- La variation interannuelle de production de matière végétale est grande au Sahel, surtout en ce qui concerne les plantes annuelles. Elle est irrégulière selon les sols. Il n'y a pas de corrélation simple entre la production d'herbe et la quantité de pluie. De nombreux autres paramètres interviennent : répartition des précipitations, intensité des pluies, importance du ruissellement, nature du sol. Le disponible fourrager est donc variable chaque année. Les mesures faites pour cette étude correspondent à une année moyenne, en ce qui concerne la production des pâturages ; 
- L'hétérogénéité naturelle des pâturages sahéliens, surtout lorsqu'ils sont dégradés, rend difficile l'évaluation précise de la production de fourrage. Les chiffres avancés ne représentent qu'une estimation de la production végétale réelle.

Rechercher une plus grande précision n'aurait guère de sens en raison de la variabilité naturelle de la production, et de la dégradation lente de la matière végétale une fois produite. En outre, les animaux manifestent en face de ces variations des ressources fourragères, un comportement qui, d'une certaine façon, tend à en équilibrer les effets.

Il faut noter qu'en raison de la superficie restreinte de cette zone d'endodromie, tous les pâturages sont exploitables facilement. Il n'existe pas de zone sous-utilisée, et l'exploitation des ressources fourragères est complète au bout du cycle annuel.

\section{RÉSULTATS}

\section{Etat actuel des pâturages et des troupeaux}

Le périmètre étudié correspond à la zone la plus chargée en bétail de tout l'Oudalan. Le pâturage manifeste cette forte exploitation animale par une dégradation assez avancée dans certains secteurs. Au dire des habitants, cette dégradation se manifeste depuis une quinzaine d'années. Elle est apparente surtout depuis les années de sécheresse de 1972 et 1973. Elle concerne plus particulièrement les glacis et les bas de versant à proximité des marigots et des zones de passage (4). En certains points intensément fréquentés comme les abords des points d'eau permanents, l'exploitation pastorale est excessive.

Cette surexploitation est particulièrement évidente à certaines saisons. Elle se traduit évidemment sur l'état des animaux qui perdent relativement beaucoup de poids en fin de saison sèche; selon les observations faites en 19761977, la perte de poids est surtout accusée entre avril et juin et elle doit dépasser 20 p. $100 \mathrm{du}$ poids de début de saison sèche. Les effets en sont donc très apparents sur les animaux, très maigres au retour des pluies et dans certains cas à la limite de l'épuisement. Toutes les classes d'âge et de sexe semblent exposées à cet amaigrissement saisonnier, mais plus particulièrement les vaches reproductrices en lactation; les jeunes en croissance présentent souvent aussi un très mauvais état.

\section{Nombre d'animaux dans le périmètre}

L'analyse des résultats de l'enquête menée en avril 1976 concernant les effectifs animaux de la zone d'endodromie considérée nous fournit les estimations présentées au tableau I ci-dessous. Les effectifs sont présentés par espèce. Les charges dues à chaque espèce sont ensuite rapportées à une même unité, l'U. B. T., Unité Bovin Tropical (l'U. B. T. est une unité pondérale de référence correspondant à un bœuf de $250 \mathrm{~kg}$ ).

TABL. $\mathrm{N}^{0} \mathrm{I}$ - Dënombrement des animaux et estimation de la charge en bétail.

\begin{tabular}{|l|r|r|r|}
\hline & Effectifs & $\begin{array}{r}\text { Nombre moyen } \\
\text { d'U.B.T./tête }\end{array}$ & $\begin{array}{c}\text { Charge en } \\
\text { U.B.T. }\end{array}$ \\
\hline Bovins & 23500 & 0,85 & 11500 \\
Caprins & 30000 & 1,15 & 2000 \\
Ovins & 7000 & 1,10 & 700 \\
Anes & 1500 & 1,3 & 500 \\
Chevaux et & 400 & 1,5 & 600 \\
chame aux & & & 15300 \\
\hline
\end{tabular}

Cette approche certes approximative permet de simplifier le raisonnement pour comparer ensuite charge en bétail et disponible fourrager. Ces estimations nous permettent, en particulier, une évaluation globale des besoins en matière sèche et en énergie sur la base des normes suivantes:

Besoins en matière sèche :

$$
6,25 \mathrm{~kg} \text { par U. B. T./jour }
$$

Besoins énergétiques :

$$
\text { 3,1 U. F. par U. B. T./jour. }
$$

Pour la population animale de la zone considérée, on obtient alors des besoins annuels arrondis à $17000000 \mathrm{U}$. F. et $33660 \mathrm{t}$ de matière sèche fourrage (voir ci-après).

\section{Disponible fourrager}

Les résultats des estimations de la production fourragère par grands types de pâturages sont reportés dans le tableau II. 
TABLEAU $\mathrm{N}^{\circ} \mathrm{II}$

\begin{tabular}{|c|c|c|c|c|}
\hline \multirow{2}{*}{$\begin{array}{l}\text { Grands types } \\
\text { de pâturages }\end{array}$} & \multicolumn{2}{|c|}{ Sup e-rficies } & \multicolumn{2}{|c|}{ Production fourragère totale } \\
\hline & en $\mathrm{km}^{2}$ & $\begin{array}{l}\text { en p.100 } \\
\text { du total }\end{array}$ & $\begin{array}{c}\text { en } t \\
\text { de M.S. }\end{array}$ & $\begin{array}{l}\text { en witlions } \\
\text { d }^{\top} \mathrm{U}, \mathrm{F} \text {. }\end{array}$ \\
\hline Pâturages sur sables & 83 & 12,9 & 10420 & 4,7 \\
\hline Pâturages sur glacis & 316 & 49,2 & 22920 & 10,3 \\
\hline Bush & 54 & 8,4 & 4090 & 1,6 \\
\hline Cordons rizicoles arborës & 18 & 2,8 & - & - \\
\hline Niares & 9 & 1,4 & 4810 & 3,5 \\
\hline Total hors cultures & $4 B O$ & 74,8 & 42240 & 20,1 \\
\hline Périmètres cultivés & 162 & 25,2 & 38800 & 17,5 \\
\hline Total & 642 & 100,0 & 81040 & 37,6 \\
\hline
\end{tabular}

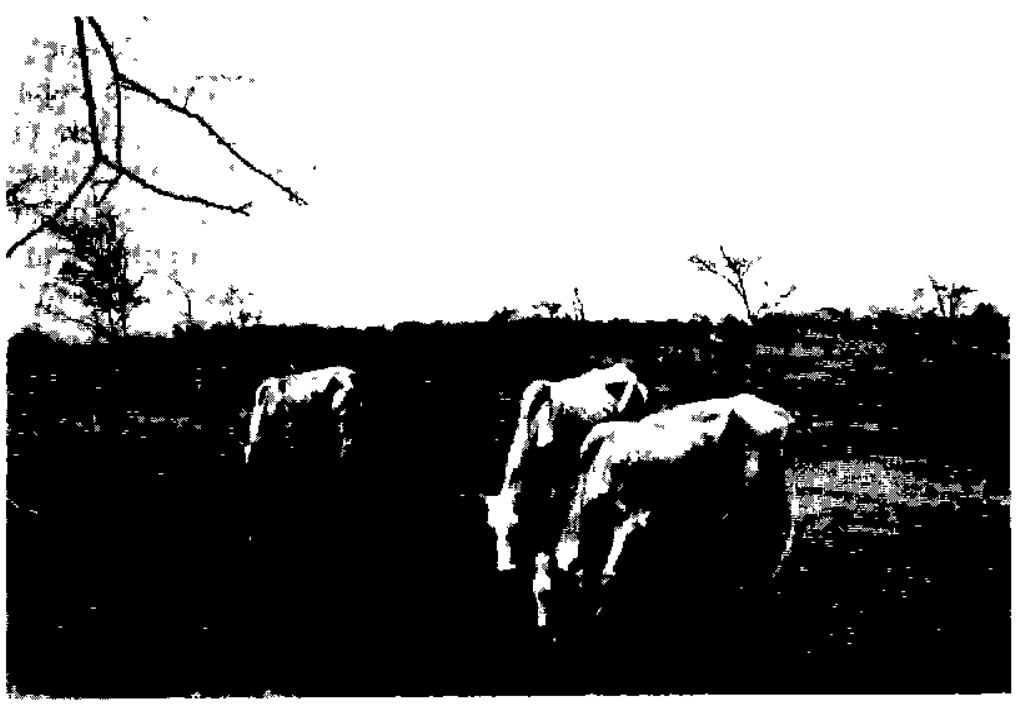

Photo $\mathbf{n}^{\circ}$ 1. - Animaux très amaigris en fin de saison sèche.

Photo $\mathrm{n}^{\circ} 2$. - Vache proche de l'épuisement.

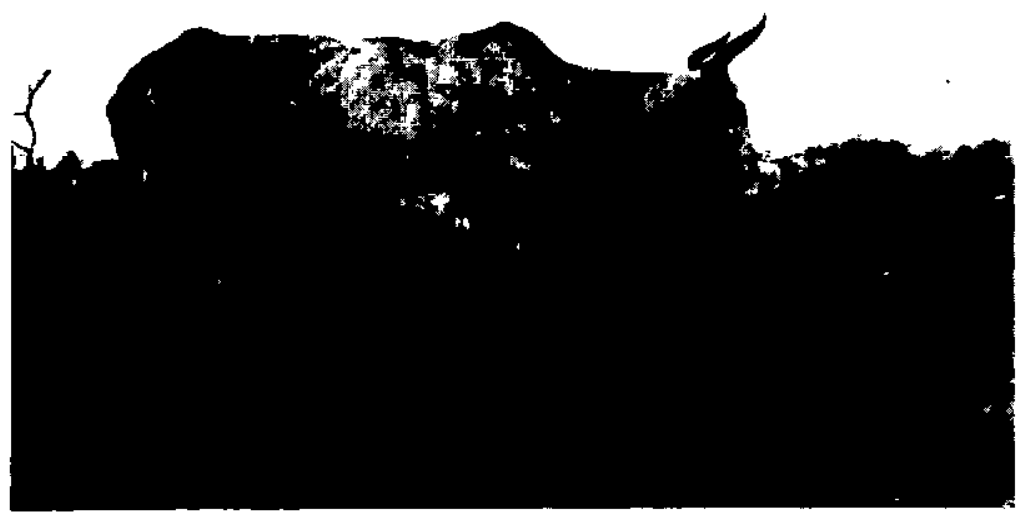


On remarque que les glacis, faibles producteurs d'herbe, sont de loin, les plus étendus. Quant aux périmètres cultivés, ils fournissent une part importante de la matière végétale produite en raison de leur extension et de la richesse de leur sol.

\section{Besoins fourragers}

On estime qu'un bovin consomme normalement par jour et par $100 \mathrm{~kg}$ de poids vif une quantité de fourrage ordinaire correspondant à $2,5 \mathrm{~kg}$ de matière sèche (soit $6,25 \mathrm{~kg}$ pour 1 U. B. T.). La quantité ingérée annuellement par un bœuf de $250 \mathrm{~kg}$ est donc de l'ordre de $2200 \mathrm{~kg}$ de matière sèche.

Les besoins énergétiques d'un bovin de $250 \mathrm{~kg}$ assurant l'entretien et la croissance ou la production de façon modérée sont proches de 3,1 U. F. par jour.

Le tableau III rassemble les besoins fourragers de l'ensemble du cheptel de la zone d'endodromie.

\begin{tabular}{|l|c|c|c|}
\hline & $\begin{array}{c}\text { Besoins } \\
\text { du chep tel }\end{array}$ & $\begin{array}{c}\text { Disponible } \\
\text { moyen }\end{array}$ & $\begin{array}{c}\text { Pourcentage } \\
\text { effectivement } \\
\text { consomené }\end{array}$ \\
\hline $\begin{array}{l}\text { Quantité de } \\
\text { fourrage en } \\
\text { to de M.S. }\end{array}$ & 33660 & 82040 & $41 \mathrm{p} \cdot 100$ \\
\hline $\begin{array}{l}\text { Unitếs four- } \\
\text { ragēres en } \\
\text { millions } \\
\text { d' U.F. }\end{array}$ & 17 & 37,6 & $45 \mathrm{p} \cdot 100$ \\
\hline
\end{tabular}

\section{DISCUSSION ET CONCLUSION}

L'évaluation qui a été proposée des besoins alimentaires du cheptel appelle quelques observations :

- L'effectif d'abord est variable avec la saison en raison de la mobilité des troupeaux d'une zone à l'autre et de la perméabilité des limites de zone ; aussi, à l'époque du dénombrement, il existe une surcharge temporaire marquée en raison de l'attraction de certains points-deau (mare d'Oursi spécialement).

- L'estimation également des besoins est approchée mais sous-estime probablement les besoins réels des petits ruminants ; pour toutes les espèces, les normes moyennes adoptées ne prévoient que de très faibles productions.

La quantité de matière végétale appétible réellement, disponible sur l'ensemble de la zone, est probablement légèrement supérieure à la valeur qui a été calculée. En effet :

- il n'a pas été tenu compte du fourrage aérien fourni par les arbres et les arbustes ;

- la quantité de résidus de culture après la récolte des épis de mil ou de sorgho a été sousestimée pour compenser la faible proportion relative de ce type de fourrage qui semble effectivement prélevée par les troupeaux. Mais il est certain aussi que cette part prélevée est variable d'un lieu à l'autre et d'une année sur l'autre selon les besoins alimentaires.

Il résulte de ces considérations que le coefficient d'utilisation des ressources fourragères par les troupeaux, qui est de 41 p. 100 , semble un maximum. La part des ressources énergétiques consommées, 45 p. 100 , est voisine bien qu'un peu supérieure. Elle peut indiquer que, pendant une partie de l'année, le fourrage n'est pas suffisamment riche pour assurer une ration énergétique suffisante, et les animaux perdent du poids.

L'évolution actuelle de la végétation à l'intérieur de la zone d'endodromie révèle que la charge annuelle actuelle de $4,2 \mathrm{ha} / \mathrm{U}$. B. T. est trop forte : le surpâturage est évident en bien des endroits. L'exploitation raisonnable du pâturage, assurant la pérennité de la végétation, correspond approximativement à la consommation de 35 p. 100 de la biomasse herbacée produite.

L'excès de charge actuel entraîne une mauvaise alimentation chronique des animaux, car ceux-ci, pressés par la faiml, sont conduits à accepter des refus de médiocre valeur. De plus, il entraîne la consommation d'une part de la végétation qui doit rester au sol pour en assurer le couvert au moment des premières tornades, et la protection des semences.

Enfin, les refus sont une source d'alimentation en cas de nécessité et sur lesquels il faut compter lorsque la croissance du pâturage aura été déficitaire une année. Ce sont ces restes qui constituent les réserves fourragères les plus sûres pour les années sèches.

En conclusion, le coefficient moyen d'utilisation par les troupeaux de la biomasse fourragère 
totale produite par les pâturages sahéliens est d'environ 35 p. 100 . Elle correspond à peu près à la valeur du tiers qui est communément utilisé pour les calculs de capacité de charge à partir des mesures de productivité des pâturages.
L'analyse et les observations proposées sur la zone d'étude semblent bien confirmer qu'il n'est pas possible de dépasser beaucoup cette norme (35 p. 100) sans surexploitation dangereuse pour le milieu et pour le cheptel.

\section{SUMMARY}

\section{Estimation test of the coefficient of herbaceous biomass utilization} by cattle in a Sahelian area

In a well delimited small Sahelian area, the authors try to evaluate the average coefficient of herbaceous biomass utilization by cattle left in this area during the year. Fodder estimation of the considered area is compared to cattle fodder need estimation after an inventory of the herbivorous domestic livestock of the area. Collected data allow to fix this coefficient at about 35 p. 100.

\section{RESUMEN}

Ensayo de evaluación del coeficiente de utilización de la biomasa herbácea por el ganado en una zona saheliana

En una pequeña región saheliana bien delimitada, los autores intentan evaluar el coeficiente medio de utilización de la biomasa vegetal herbácea apetecible por el conjunto del ganado presente durante el año, comparando la evaluación de las reseryas forrajeras de todo el perimetro y la de las necesidades forrajeras del ganado despuès de la cuenta de los animales domésticos herbivoros que frecuentan esta región. Según las observaciones recogidas, el coeficiente llega a nuos 35 p. 100.

\section{BIBLIOGRAPHIE}

1. BARRAL (H.). Mobulité et cloisonnement chez les éleveurs du Nord de la Haute-Volta : les zones dites «d'endodromie » pastorales. Sémin. Int. Pastoralisme, Alger, avril 1974, $17 \mathrm{p}$.

2. BILLE (J. C.). Etude de la production primaire nette d'un écosystème sahélien. Paris, thèse d'Etat, 18 octobre $1976,82 \mathrm{p}$.

3. BOUDET (G.). Manuel sur les pâturages tropicaux et les cultures fourragères. Paris, Minist. Coopération, 1975, 254 p. (Coll. I. E. M. V. T., Manuels et Précis d'Elevage, $n^{0} 4$ ).

4. DE WISPELAERE (G.), TOUTAIN (B.). Estimation de l'évolution du couvert végétal en 20 ans, consécutivement à la sécheresse dans le Sahel voltaïque. Photo-interprétation, 1976, 15 (3) fasc. 2.

5. LIIOSTE (P.). Rapport sur l'étude zootechnique de l'action concertée pour l'étude des conditions physiques, biologiques et humaines de la lutte contre l'aridité dans l'Oudalan.

6. TOUTAIN (B.). Notice de la carte des ressources fourragères à $1 / 50000$ suivie de commentaires sur la répartition des pâturages et leur potentiel de charge. Action Complémentaire Concertée D. G. R.S. T./ O. R. S. T. O. M. Paris, G.E.R.D.A.T./I.E.M.V.T., 1976,61 p. +6 p., 1 carte au $1 / 50000$.

\section{ERRATUM}

In : article ILEMOBADE (A. A.), LEEFLANG (P.). Epidemiology of heartwater in Nigeria. Rev. Elev. Méd. vét. Pays trop., 1977, 30 (2) : 149.

$2^{e}$ paragraphe du résumé : au lieu de : «la transmission trans-stadiale n'a pu être démontrée $»$.

Lire :

« la transmission trans-stadiale du parasite a toujours réussi ; par contre une transmission transoyariale n'a pu être démontrée $\%$. 